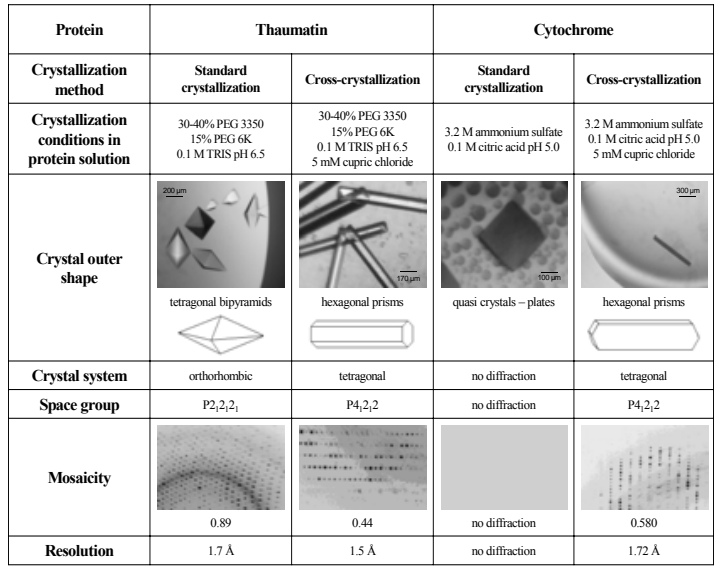

This work is supported by grants MSM6007665808 and LC06010 of the Ministry of Education of the Czech Republic and Institutional research concept AVOZ60870520 of Academy of Sciences of the Czech Republic to I.K.S.

\section{MS06 P07}

High-Temperature Crystallization of Thermostable T1 Raja Noor Zaliha Raja Abdul Rahman ${ }^{l}$, Thean Chor Leow $^{l}$, Abu Bakar Salleh ${ }^{l}$,Mahiran Basri ${ }^{2}$ and Mohd. Basyaruddin Abdul Rahman'.

Enzyme and Microbial Technology Research Group, ${ }^{I}$ Faculty of Biotechnology and Biomolecular Sciences, ${ }^{2}$ Faculty of Science, Universiti Putra Malaysia, 43400 UPM Serdang, Selangor, Malaysia

E-mail: rnzaliha@biotech.upm.edu.my

Keywords: thermostable lipase, high temperature, protein crystallization

The gene encoding thermostable T1 lipase secreted by Geobacillus sp. strain T1 was overexpressed in a prokaryotic system. Preliminary crystallization was conducted with crystal screen and crystal screen II through a sitting drop vapor diffusion method with $0.5 \mathrm{mg} / \mathrm{mL}$ purified $\mathrm{T} 1$ lipase at $16^{\circ} \mathrm{C}$. Crystallization at $16^{\circ} \mathrm{C}$ using formulation 21 of crystal screen II at $2.5 \mathrm{mg} / \mathrm{mL}$ yielded bigger and more defined crystals. Good crystals could easily be obtained as the temperature was increased further while retaining other conditions. In fact, crystallization of $\mathrm{T} 1$ lipase is still possible at $60^{\circ} \mathrm{C}$ and this is new in lipase crystallization.

\section{MS06 P08 \\ Structural investigation of human thrombomodulin domains. Lou KL, Chen KT and Wu HL. Graduate Institute of Oral Biology, College of Medicine, National Taiwan University, Taipei, Taiwan. \\ Institute of Biochemistry and Molecular Biology, College of Medicine, National Cheng-Kung University, Tainan, Taiwan.}

Thrombomodulin (TM) is a multifunction glycoprotein expressed on the endothelial cell surface. This glycoprotein is structurally organized into 5 distinct domains. From the $\mathrm{C}$ - to $\mathrm{N}$-terminus, TM has a short cytoplasmic tail on the intracellular side of plasma membrane, a transmembrane helical segment, and the extracellular part containing a serine/threonine-rich region, EGF-like repeats, as well as an N-terminal C-type lectin domain. Each of the distinct domains has different biological functions that impact on coagulation, fibrinolysis, inflammation, cell Adhesion, and cell proliferation. To understand how this single molecule may play different important roles through distinct domains, we commenced the crystal structural analysis of TM with domain variants combination. Various domains of TM were constructed and expressed in Pichia Pastoris: (i) the TMD-1 construction contains the C-type lectin domain, (ii) the TMD-23 construction contains the EGF-like repeats and the serine/threonine-rich region, and (iii) the TMD-123 construction contains all the extracellular domains. Crystallization screening indicated successful conditions for TMD-23 and potential ones for TMD-1 requiring optimization.

\section{MS06 P09}

Pulsed, high-voltage, inhomogeneous electric fields improve nucleation and crystal growth

Uwe H. Sauer, UCMP, Umeå University, SE-901 87

Umeå , Sweden. E-mail: uwe.sauer@ucmp.umu.se

In order to avoid multiple nucleations, leading to showers of micro crystals, we investigate the effect of an highvoltage, inhomogeneous electrical fields on the protein concentration in crystallization drops.

Our results indicate that the high voltage, inhomogeneous E-field increases the probability, for nucleation from lower protein concentration. Temporally controlled exposure of proteins in sitting or hanging drops to strong inhomogeneous E-fields of $3000-5000 \mathrm{~V}$ leads to nucleation even in dilute protein solutions. Switching the E-field off allows for the formation of large single crystals.

[1] Taleb M., Didierjean C., Jelsch C., Mangeot J.P., Capelle B. and Aubry A.. J. Cryst. Growth, 1999, 200, 575.

[2] Taleb M., Didierjean C., Jelsch C., Mangeot J.P., and Aubry A.. J. Cryst. Growth, 2001, 232, 250.

\section{MS06 P10}

Dynamic screening experiments to maximize hits for protein crystallization Naomi E. Chayen, Sahir Khurshid and Lata Govada Department of Bio Molecular Medicine Division of Surgery, Oncology, Reproductive Biology and Anaesthetics, Faculty of Medicine, Imperial College Londo, $n$ Sir Alexander Fleming Building, London SW7 2AZ, UK

E-mail: n.chayen@imperial.ac.uk

\section{Keywords: Screening, Vapour diffusion, Nucleation}

In the first step of crystallization screening, the protein is exposed to a wide variety of reagents at different concentrations. Once a "hit" deemed to be conducive to crystallization is identified, parameters such as precipitant concentration, $\mathrm{pH}$ and temperature are used to produce crystals suitable for analysis by X-ray diffraction. Crystals, crystalline precipitate and phase separation are usually considered leads that are worth pursuing. Clear drops are mostly disregarded. This poster presents a screening technique that makes use of clear drops. Clear drops are subjected to evaporation with the aim of driving them to supersaturation. The findings reported bring a new dimension to screening and open up the scope for utilizing a potential wealth of crystallization conditions that are currently being ignored. Furthermore, this technique enables the utilization of far less protein sample as well as obtaining the 'hits' in shorter times. 